\title{
Notch inhibition restores TRAIL-mediated apoptosis via AP1-dependent upregulation of DR4 and DR5 TRAIL receptors in MDA-MB-231 breast cancer cells
}

\author{
PATRIZIA PORTANOVA ${ }^{1^{*}}$, ANTONIETTA NOTARO ${ }^{2 *}$, ORNELLA PELLERITO $^{3}$, \\ SELENIA SABELLA $^{2}$, MICHELA GIULIANO ${ }^{2 * *}$ and GIUSEPPE CALVARUSO ${ }^{2 * *}$
}

\author{
${ }^{1}$ Dipartimento di Medicina traslazionale, Università del Piemonte Orientale, Novara; ${ }^{2}$ Dipartimento di Scienze e Tecnologie \\ Biologiche Chimiche e Farmaceutiche, Università di Palermo, Palermo, Italy; ${ }^{3}$ Laboratory of Cellular and \\ Developmental Genetics, Department of Molecular Biology, Medical Biochemistry and Pathology, \\ University of Laval, Québec, Canada
}

Received January 9, 2013; Accepted March 14, 2013

DOI: 10.3892/ijo.2013.1945

\begin{abstract}
Notch is a family of transmembrane receptors whose activation through proteolytic cleavage by $\gamma$-secretase targets genes which participate in cell development, differentiation and tumorigenesis. Notch signaling is constitutively activated in various cancers, including breast cancer and its upregulation is usually related with poor clinical outcomes. Therefore, targeting Notch signaling with $\gamma$-secretase inhibitors (GSIs) is considered a promising strategy for cancer treatment. We report that the $\gamma$-secretase inhibitor-I (GSI-I) sensitizes human breast cancer cells to apoptosis mediated by tumor necrosis factor-related apoptosis-inducing ligand (TRAIL). The antiproliferative GSI-I/TRAIL synergism was stronger in ER-negative MDA-MB-231 breast cancer cells compared with ER-positive MCF-7 cells. In MDA-MB-231 cells, GSI-I treatment induced upregulation of DR4 and DR5 TRAIL receptors. This effect seemed to be related to the activation of the transcription factor AP1 that was a consequence of Notch inhibition, as demonstrated by Notch-1 silencing experiments. Combined treatment induced loss of the mitochondrial transmembrane potential and activation of caspases. GSI-I alone and/or GSI-I/TRAIL combination also induced a significant decrease in the levels of some survival factors (survivin, c-IAP-2, Bcl- $\mathrm{x}_{\mathrm{L}}, \mathrm{Bim}_{\mathrm{EL}}$ and $\mathrm{pAKT}$ ) and upregulation of pro-apoptotic factors $\mathrm{Bim}_{\mathrm{L}}, \mathrm{Bim}_{\mathrm{S}}$ and Noxa,
\end{abstract}

Correspondence to: Professor Giuseppe Calvaruso, Dipartimento di Scienze e Tecnologie Biologiche Chimiche e Farmaceutiche, Università di Palermo, Via del Vespro 129, I-90127 Palermo, Italy

E-mail: giuseppe.calvaruso@unipa.it

*Contributed equally as first authors

${ }^{* *}$ Contributed equally as senior authors

Key words: notch signaling, $\gamma$-secretase inhibitor-I/tumor necrosis factor-related apoptosis-inducing ligand combined treatment, apoptosis, breast cancer cells, AP-1 enhancing the cytotoxic potential of the two drugs. Taken together, these results indicate for the first time that GSI-I/ TRAIL combination could represent a novel and potentially effective tool for breast cancer treatment.

\section{Introduction}

It is well known that Notch signaling plays fundamental roles in the cell, including the maintenance of stemness, the regulation of cell proliferation, differentiation and apoptosis during the cell development (1). Notch is a transmembrane heterodimeric receptor present in humans as four distinct members (Notch 1-4). Following the binding of the specific ligand, Notch is activated by a cascade of $\alpha$ and $\gamma$-secretase-dependent proteolytic cleavages and its intracellular domain Notch-IC (NIC) translocates into the nucleus becoming transcriptionally active (2). It has been demonstrated that many different cancers are characterized by the constitutive activation of Notch pathway induced by genetic alterations such as chromosomal amplification or translocation at the Notch receptor loci (3). Dysregulation in Notch signaling has been found in various subtypes of breast, colon and prostate carcinomas, melanoma and haematological malignancies (4-8). Thus, its pathway could represent a potential therapeutic target in cancer treatment (9). In particular, since all Notch receptors are activated by $\gamma$-secretase, the inhibitors of this enzyme (GSIs) have attracted increasing interest (10). GSIs were firstly employed in the treatment of Alzheimer's disease to prevent amyloid precursor protein (APP) cleavage and the consequent release of amyloid $\beta$-peptide (11). More recently, it has been observed that GSIs also possess the ability to induce growth arrest and/or apoptosis in some tumor cell lines while other tumor cells were resistant to the molecules (12).

Similarly to $\gamma$-secretase inhibitors, also tumor necrosis factor-related apoptosis-inducing ligand (TRAIL), a member of the superfamily of tumor necrosis factor, is capable of inducing apoptosis in a wide variety of cancer cells showing an anticancer potential (13-15). TRAIL-dependent apoptosis is triggered by the binding of TRAIL to its specific DR4 and DR5 receptors which results in the interaction with the adaptor molecule FADD and 
in the recruitment and cleavage of the initiator caspase- 8 with the consequent activation of executioner protease cascade (16). However, it has been reported that a number of malignant cells are resistant or develop resistance to TRAIL-induced signaling. This is frequently owed to the loss of DR4 and DR5 receptors on cell surface (17).

A good strategy to obtain therapeutic advantages in cancer cell death could be to find combinations of different compounds which act in synergistic manner in order to overcome cell resistance and/or allow a dose reduction to decrease the side-effects. Many authors demonstrated that TRAIL signaling can be restored in TRAIL resistant cancer cells by different classes of compounds, such as cannabinoids (18), inhibitors of proteasome (19) or of histone deacetylases (20). Thus, the combination of these compounds with recombinant TRAIL results in synergistic cytotoxic effects. The mechanisms underlying these effects involve the activation of different biochemical pathways which have been often clarified even if, in other cases, the molecular elucidation of the events remains obscure. On the other hand, a limit of the employment of GSIs in therapy is represented by their adverse effects in normal cells probably due to the wide variety of cellular functions of $\gamma$-secretase activity (21). To overcome this problem, the inhibitors of $\gamma$-secretase have been recently employed in combination with other compounds with the aim to identify a therapeutic window in which the dosage is sufficient to suppress Notch signaling without affecting normal cell functions. Promising results have been obtained in haematological cancer malignancies by employing GSIs in combination with Hedgehog and Wnt inhibitors (22) as well as with proteasome inhibitors (23).

In this study we investigated whether GSI-I, one of the most efficacious $\gamma$-secretase inhibitors, is able to revert TRAIL resistance of two breast cancer MDA-MB-231 and MCF-7 cell lines. The results indicate that the association of GSI-I/TRAIL induces strong cytotoxic synergistic effects in MDA-MB-231 cells which were related to overexpression of DR4 and DR5 TRAIL receptors. This was probably mediated by the activation of the transcription factor AP1 which was dependent on Notch cleavage inhibition. We suppose that this new combined treatment can be useful to improve the therapeutic efficacy in TRAIL-resistant breast cancer cells.

\section{Materials and methods}

Reagents. $\gamma$-secretase inhibitor I (Z-LLNle-CHO) (GSI-I) was from Calbiochem (San Diego, CA, USA). TRAIL signaling has been activated using a soluble human recombinant TRAIL/ APO2L (PeproTech EC Ltd, London, UK) containing the residues of amino acids from 114 to 281 of natural TRAIL. z-DEVD-cho was purchased from Promega (Milan, Italy). Stock solutions were prepared in dimethyl sulfoxide (DMSO) and opportunely diluted in culture medium. The final concentration of DMSO never exceeded $0.1 \%$, a percentage which was not toxic and did not interfere with cell growth.

Cell cultures and viability and death assays. Human breast cancer estrogen receptor (ER)-negative MDA-MB-231 and ER-positive MCF-7 cells were cultured in Dulbecco's modified Eagle's medium high glucose (DMEM/HIGH) supplemented with $2.0 \mathrm{mM}$ L-glutamine, $10 \%$ (v/v) heat-inactivated fetal bovine serum (FBS) and antibiotic-antimycotic solution (100 U/ml penicillin, $100 \mu \mathrm{g} / \mathrm{ml}$ streptomycin and $250 \mathrm{ng} /$ $\mathrm{ml}$ amphotericin B, Sigma Chemical). Non-essential amino acid solution (1.0 mM) was added only in MDA-MB-231 cell culture medium. Cells were maintained at $37^{\circ} \mathrm{C}$ in a humidified atmosphere containing $5 \% \mathrm{CO}_{2}$. For the experiments, cells were seeded at $60-70 \%$ confluence, unless otherwise indicated and allowed to adhere overnight. Control cells were cultured in the presence of vehicle alone.

Cell viability was determined by 3-[4,5-dimethylthiazolyl-2] 2,5-diphenyltetrazolium bromide assay (MTT, Sigma Chemical) as previously reported (24). Cell survival was reported as a percentage of the control value referred to vehicle-treated cells.

In order to ascertain the presence of condensed chromatin and apoptotic bodies, cells were stained with Hoechst 33258 dye. Cells, seeded in 96-well plates, were fixed in 3:1 methanol/acetic acid for $10 \mathrm{~min}$ at room temperature, washed in phosphate-buffered saline (PBS) and stained for $30 \mathrm{~min}$ in PBS containing $40 \%$ paraformaldehyde and $10 \mu \mathrm{g} / \mathrm{ml}$ Hoechst 33258. After washing in PBS nuclear morphology was observed under a fluorescence microscope equipped with an automatic photomicrograph system (Leica, Germany).

Flow cytometric analysis. Apoptotic cells were quantified by measuring the externalized phosphatidylserine residues by using Annexin V-FITC/propidium iodide kit (BD Biosciences, San Diego, CA, USA) following the manufacturer's instructions. After treatment, cells were collected, washed with ice-cold PBS and suspended in a binding buffer at a concentration of $10^{6}$ cells $/ \mathrm{ml}$. Flow cytometric analysis was carried out as previously reported (18).

Mitochondrial transmembrane potential $(\Delta \psi \mathrm{m})$ dissipation was measured by using 3,3-dihexyloxacarbocyanine ( $\mathrm{DiOC}_{6}$ ), a lipophilic fluorochrome which exclusively emits within the spectrum of green light and accumulates in the mitochondrial matrix under the influence of $\Delta \psi \mathrm{m}$. Cells were incubated with $40 \mathrm{nM} \mathrm{DiOC}_{6}$ for $20 \mathrm{~min}$ at $37^{\circ} \mathrm{C}$, washed with PBS and analyzed by flow cytometry with excitation and emission setting of 488 and $525 \mathrm{~nm}$, respectively, using Expo 32 software. The percentage of cells showing a lower fluorescence, reflecting the loss of $\Delta \psi \mathrm{m}$, was determined by comparison with untreated controls.

Carbonyl cyanide m-chlorophenylhydrazone $(50 \mu \mathrm{M})$, a protonophore that completely de-energizes mitochondria by dissipating the transmembrane potential, was used as a positive control for maximum $\Delta \psi \mathrm{m}$ disruption (data not shown).

Western blot analysis. Western blot analysis was performed as previously reported (25). Protein bands were visualized using chemiluminescence or alkaline phosphatase staining. Bands were quantified by densitometric analysis using ImageJ software (NIH). The correct protein loading was verified by means of both red Ponceau staining and immunoblotting for actin. All the antibodies used were purchased from Santa Cruz Biotechnology (Santa Cruz, CA, USA), except for anti-caspase-8, -9, -3 and Bim (Cell Signaling, Beverly, MA, USA) and anti-DR5 and -DR4 (ProSci, Poway, CA, USA).

Quantitative RT-PCR analysis. Total RNA was extracted from MDA-MB-231 cells using RNeasy Mini kit (Qiagen, 
Valencia, CA, USA) and treated with DNase I using the RQ1 RNase free DNase (Promega, Madison, WI, USA). The single-stranded cDNA was synthesized by the GeneAmp kit for reverse transcriptase-polymerase chain reaction (Perkin-Elmer, Foster City, CA, USA). Each cDNA sample (5 $\mu \mathrm{l})$ was amplified using iQ SYBR-Green Supermix (Bio-Rad, Hercules, CA, USA) according to the manufacturer's instructions. The following primers were used: $D R 4$, sense 5'-AGAACGTCCT GGAGCCTGTAAC-3', antisense 5'-ATGTCCATTGCCTG ATTCTTTGTG-3' (Ta, 63 $\left.{ }^{\circ} \mathrm{C}\right)$; DR5, sense 5'-GCACTCACT GGAATGACCTC-3', antisense 5'-GCCTTCTTCGCACTG ACAC-3' (Ta, 55 $\left.{ }^{\circ} \mathrm{C}\right)$; GAPDH, sense 5'-TGACATCAAGAAGG TGGTGA-3', antisense 5'-TCCACCACCCTGTTGCTGTA-3' $\left(\mathrm{Ta}, 55^{\circ} \mathrm{C}\right)$. All reactions were performed in triplicate. Linear range of a standard curve of serial dilutions was checked for each PCR. The relative quantification of DR4 and DR5 gene expression was evaluated after normalization with GAPDH gene as endogenous control. Data processing and statistical analysis were performed by using iQ5 cycler software.

Gene silencing using siRNA. Small interfering RNAs (siRNAs) against Notch-1 (siNotch-1) and scrambled siRNA (siScr), used as a negative non-silencing control, were purchased from Dharmacon RNA Technologies (Chicago, IL, USA). Notch-1 sequence (5'-AAGTGTCTGAGGCCAGCAAGA-3') was as reported by Rizzo et al (26). Cells $\left(2 \times 10^{5}\right)$ were plated in a 6 -well plate and cultured in antibiotic-free DMEM/HIGH supplemented with $2.0 \mathrm{mM}$ L-glutamine and $1.0 \mathrm{mM}$ non-essential amino acid solution for $24 \mathrm{~h}$ before the transfection to reach about $50 \%$ confluence. Then cells were transfected with $50 \mathrm{nM}$ siRNA against Notch-1 in the presence of $2.5 \mu \mathrm{l}$ lipofectamine 2000 (Invitrogen, Carlsbad, CA, USA), in a final volume of $1 \mathrm{ml}$ serum-free DME/HIGH medium. The reaction was stopped after $6 \mathrm{~h}$ of treatment replacing the medium with fresh $10 \% \mathrm{FBS}$ supplemented medium, and after $24 \mathrm{~h}$ cells were treated with TRAIL as indicated.

Statistical analysis and evaluation of synergy. Cell viability data were expressed as the mean \pm SE and evaluated by Student's t-test. Differences were considered significant when the p-values were $<0.05$. The median-effect method, originally described by Chou and Talalay (27), was employed to quantify the effects of drug combination and to evaluate eventual synergistic actions as previously reported (18). A CI value significantly $<1$ indicates synergy, a CI not significantly different from 1 indicates addition, and a CI significantly $>1$ indicates antagonism. Synergy occurs when the combination of two agents has a therapeutic effect greater than the effect of each drug.

\section{Results}

Effects of GSI-I/TRAIL combined treatment in MDA-MB-23I and MCF-7 breast cancer cells. First, the antiproliferative effects of GSI-I in combination with TRAIL were examined in two breast cancer cell lines with different estrogen receptor profiles. The dose-response curves of the effects exerted by the compounds, used as a single agent or in combination, on cell viability are reported in Fig. 1A. GSI-I at a range of doses of $0.25-1 \mu \mathrm{M}$ for $24 \mathrm{~h}$ was almost ineffective in both the two cell lines while when used at the highest concentration
$(2.5 \mu \mathrm{M})$ caused a reduction in cell viability of about $40 \%$ in MDA-MB-231 cells. The addition of $25 \mathrm{ng} / \mathrm{ml}$ TRAIL clearly potentiated the effects of GSI-I on MDA-MB-231 cells. Indeed, TRAIL combination with 1 or $2.5 \mu \mathrm{M}$ GSI-I reduced cell viability of about 30 and $15 \%$, respectively. Treatment of cells with TRAIL alone did not induce any cytotoxic effect. The addition of $100 \mu \mathrm{M}$ z-DEVD, a specific inhibitor of caspase-3, to co-treated cells induced a reduction in the cytotoxicity of about $50 \%$, indicating that GSI-I/TRAIL combination triggered a caspase-dependent cell death. In MCF-7 cells the addition of TRAIL to GSI-I treated cells induced modest effects and the reduction of cell viability was only of about $25 \%$ at the highest GSI-I concentration. Also in this case, the addition of z-DEVD reduced the effect of combined treatment (Fig. 1A).

To evaluate whether cell growth inhibition induced by GSI-I/TRAIL combination was due to a synergistic effect we analyzed cell growth inhibition curves by using the median effect method of Chou and Talalay (27). Cells were treated for $24 \mathrm{~h}$ with different doses of the two compounds employed alone or in combination at fixed molar ratios in order to obtain the concentrations reducing cell viability of 25,50 and $75 \%$. Data were used to calculate the combination index (CI) at the different fractional effects. Analysis of CI always showed values $<1.0$ indicating clear synergistic interactions between GSI-I and TRAIL (Fig. 1B). Since the effects were more evident in MDA-MB-231 than in MCF-7 cells, most of the successive experiments were performed in MDA-MB-231 cells employing $1 \mu \mathrm{M}$ GSI-I and $25 \mathrm{ng} / \mathrm{ml}$ TRAIL individually or in combination.

Then, we examined the effects of the two compounds on cell morphology by means of light microscopy. As shown in Fig. 1C (top panel) after treatment with GSI-I/TRAIL combination for $24 \mathrm{~h}$ cells appeared rounded, fragmented and floated in the medium, while the two drugs, employed separately, did not induce any significant effect. In the same experimental conditions, fluorescence microscopy analysis of nuclei stained with Hoechst 33258 evidenced a marked increase in the number of cells with condensed and fragmented nuclei, a typical apoptotic feature (Fig. 1C, middle panel).

To quantify early apoptotic effects, we analyzed the externalisation of phosphatidylserine on cell plasma membranes by means of Annexin V/propidium iodide staining. As shown in Fig. 1C (bottom panel), following GSI-I/TRAIL combined treatment for $6 \mathrm{~h}, 22.6 \%$ of MDA-MB-231 cells resulted Annexin $\mathrm{V}$ positive/propidium iodide negative, while no effect was observed in cells treated with the compounds employed separately.

GSI-I sensitizes MDA-MB-231 cells to TRAIL-induced apoptosis by upregulation of DR4 and DR5 death receptors. It is well known that in many experimental systems TRAIL actions are dependent on the presence of its specific death receptors DR4 and DR5 on cell membrane (28). Western blot analysis showed that MDA-MB-231 cells express very small amounts of these receptors and this could explain TRAIL resistance (Fig. 2A). The addition of $1 \mu \mathrm{M}$ GSI-I time-dependently enhanced the expression levels of both DR4 and DR5 which reached the maximum at $16 \mathrm{~h}$ of treatment (Fig. 2A). The increase of these receptors was a consequence of a new protein synthesis as demonstrated by real-time quantitative RT-PCR for both DR4 and DR5 mRNAs (Fig. 2B). In particular, the GSI-I effect on 
A
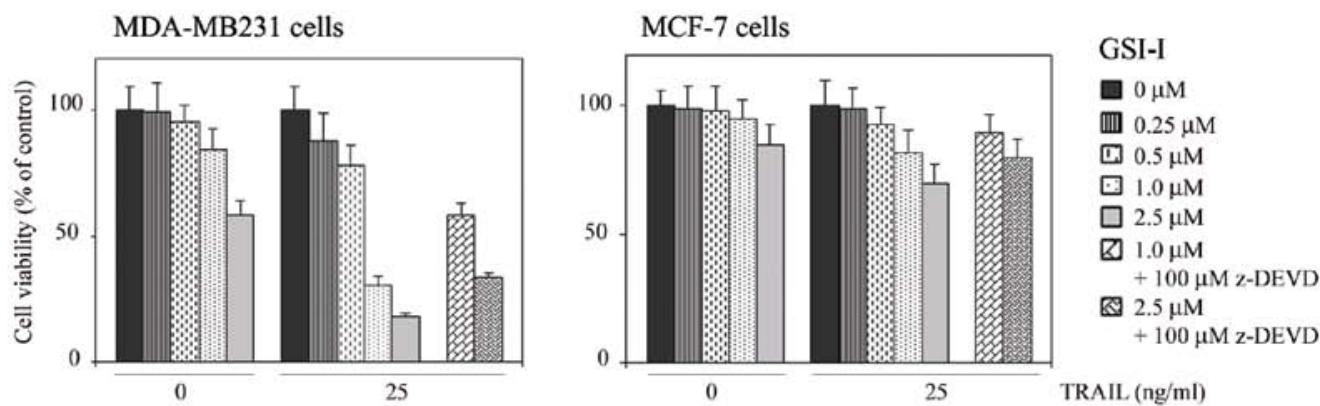

B

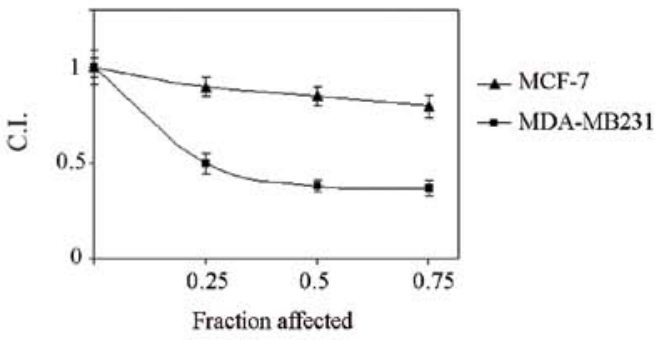

$\mathrm{C}$

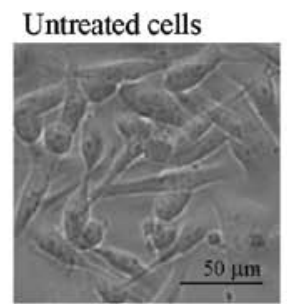

TRAIL
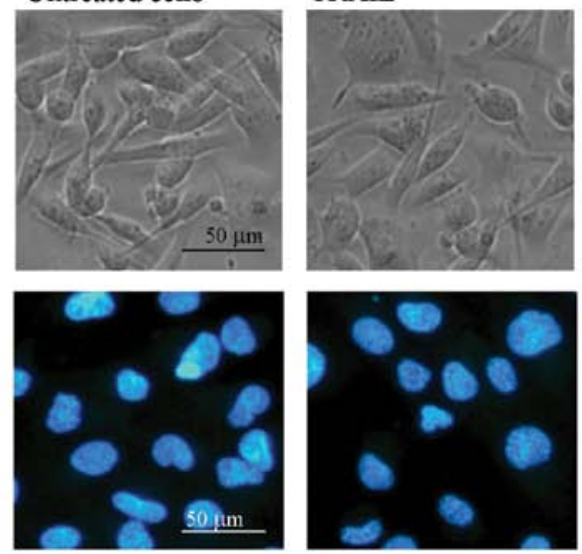

GSI-I

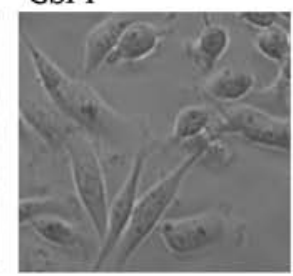

GSI-I/TRAIL
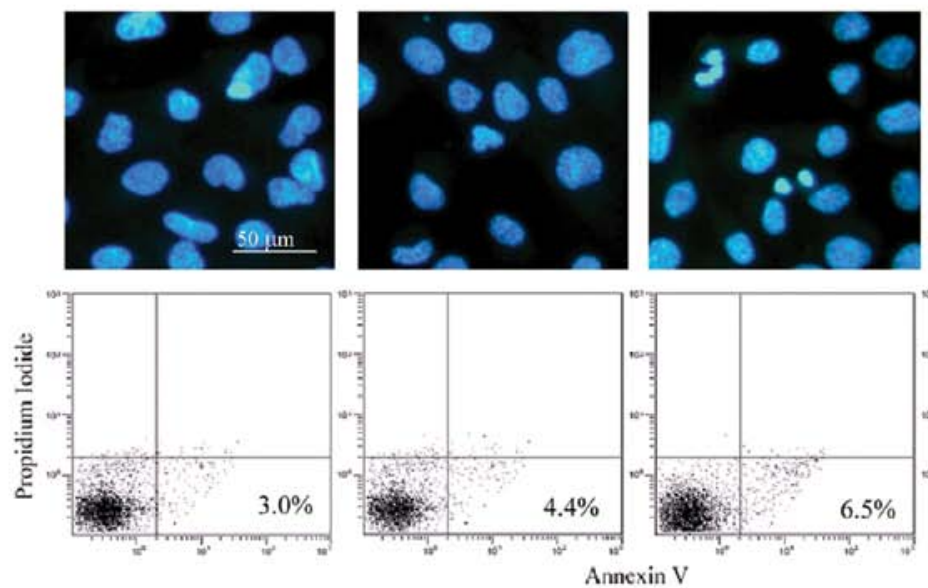
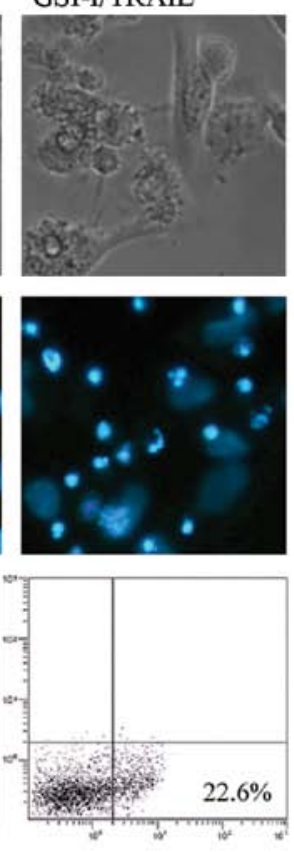

Figure 1. GSI-I/TRAIL combined treatment induces apoptotic cell death in breast cancer cells. (A) Cytotoxic effects exerted on MDA-MB-231 and MCF-7 cells by GSI-I employed alone or in combination with recombinant TRAIL. The effect of caspase-3 inhibitor z-DEVD is also shown. After treatment for $24 \mathrm{~h}$ cell viability was evaluated by MTT assay as reported in Materials and methods. (B) Synergistic effects exerted by combinations of GSI-I with TRAIL. Cells were treated for $24 \mathrm{~h}$ with the two compounds at concentrations which changed in a fixed ratio. At the end cell viability was measured by MTT assay and data were employed to calculate combination index (CI) at the different fractional effects $(\mathrm{P}<0.05)$. (C) Apoptotic effects induced by treatment of MDA-MB-231 cells with $1 \mu$ M GSI-I and/or $25 \mathrm{ng} / \mathrm{ml}$ TRAIL for $24 \mathrm{~h}$. Apoptotic morphology was evaluated by light microscopy (top panel) and after staining of the cells with Hoechst 33258 (middle panel), magnification, x200. Annexin V positive cells were quantified by flow cytometric analysis after double staining of cells with Annexin V and propidum iodide at $6 \mathrm{~h}$ of treatment (bottom panel). Results are representative of three independent experiments.

both mRNAs was evident at $8 \mathrm{~h}$ and after $16 \mathrm{~h}$ of treatment the increase in mRNAs induced by GSI-I was more than 2-fold with respect to the control value. Similar effects on DR4 and DR5, both at protein and mRNA level, were observed after treatment with GSI-I/TRAIL combination while treatment with $25 \mathrm{ng} / \mathrm{ml}$ TRAIL alone was ineffective (Fig. 2B).

GSI-I-dependent DR4 and DR5 increase triggered TRAIL-induced activation of extrinsic apoptotic pathway. 
MDA-MB 231 cells

A
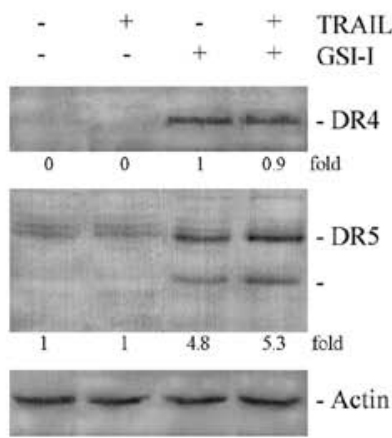

$\mathrm{C}$
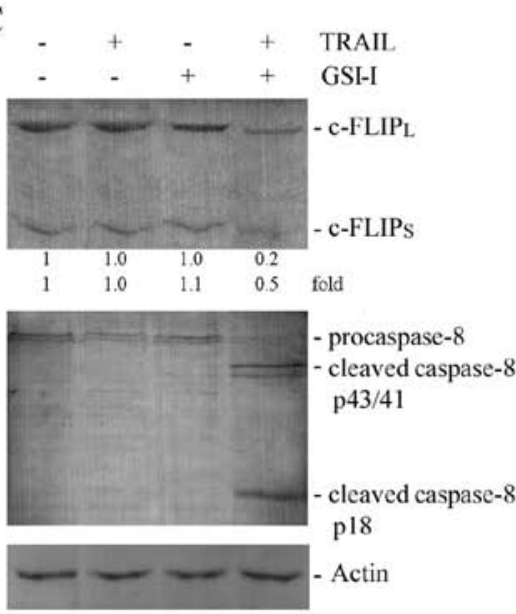

MCF-7 cells

E

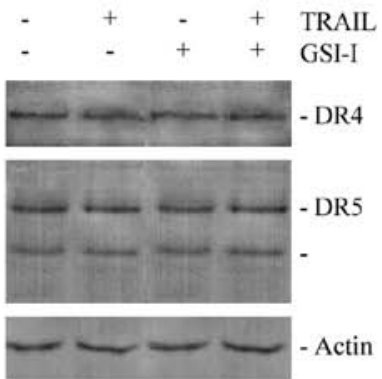

B
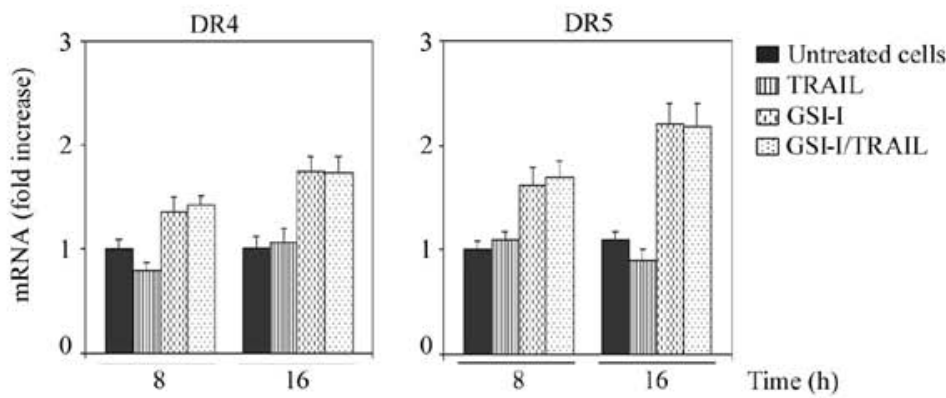

D
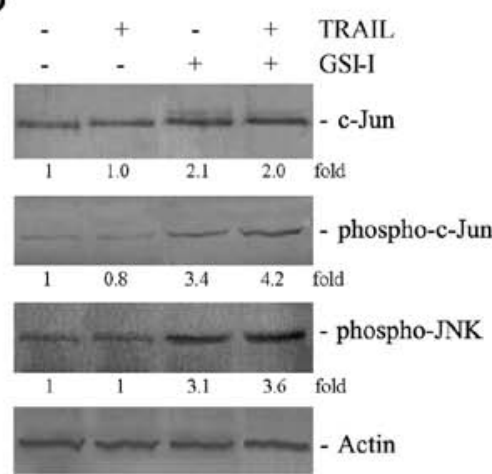

Actin

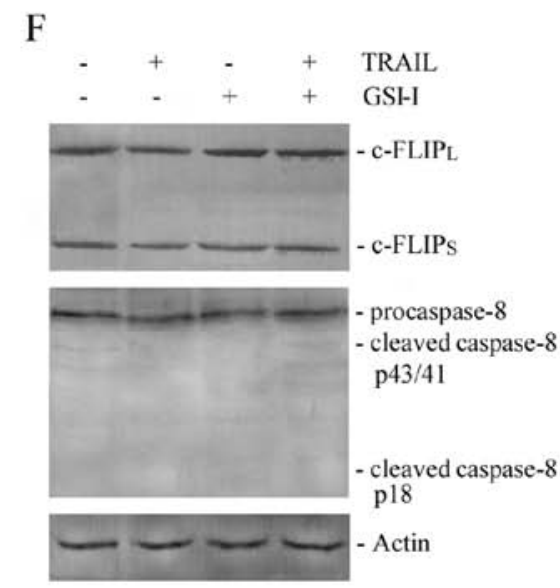

Figure 2. GSI-I stimulates the activation of TRAIL signaling and the activation of c-jun pathway in human MDA-MB-231 cells. Western blot analysis of TRAIL receptors, c-FLIP, procaspase-8 and c-jun pathway in MDA-MB-231 (A, C and D) or MCF-7 (E and F) cells. Cells were treated for $16 \mathrm{~h}$ with GSI-I and TRAIL employed alone or in combination. Western blot analyses were performed using specific antibodies as indicated. Actin blots were included to show equal protein loading for all of the samples. B reports real-time RT-PCR analysis of DR4 and DR5 mRNAs performed in MDA-MB-231 cells treated with the compounds as reported in Materials and methods. Results are representative of three independent experiments.

Western blot analysis demonstrated that GSI-I/TRAIL combined treatment for $16 \mathrm{~h}$ led to the activation of caspase- 8 evidenced as a marked decrease in the procaspase form with the concomitant appearance of the active cleaved forms. Combined treatment also induced a clear reduction of both the long and short forms of FLICE inhibitory protein (cFLIP), the main 
inhibitor of caspase-8 (Fig. 2C). No significant effects on the level of these factors were observed in cells treated with the two compounds employed separately.

Based on the above reported data, we performed experiments to investigate the mechanism whereby GSI-I induces DR4 and DR5 upregulation in MDA-MB-231 cells. It has been suggested that the transcription factor AP1 is responsible for the synthesis of DR4 and DR5 mRNAs (29). To investigate the involvement of AP1 in GSI-I and/or GSI-I/TRAIL effects, we analyzed the level of c-Jun, the main component of AP1. Fig. 2D shows that in MDA-MB-231 cells incubated in the presence of GSI-I or GSI-I/TRAIL for $16 \mathrm{~h}$ both the levels of c-Jun and its active phosphorylated form, evidenced by means of a specific antibody, were clearly increased. Since the phosphorylation and activation of c-Jun depends on the activity of phospho-JNK, we performed western blot analysis to verify whether treatment of MDA-MB-231 cells with GSI-I modulated the level of this kinase. The results indicated a significant increase in the band corresponding to phospho-JNK in cells treated with GSI-I or GSI-I/TRAIL combination.

It has been reported that MCF-7 cells express high level of DR5 TRAIL receptor even if they result resistant to TRAIL action owing to the formation of an inhibitory complex consisting of DR5, FADD, caspase-8 and c-FLIP $(30)$. Parallel experiments carried out in MCF-7 cells confirmed the presence of high levels of both DR4 and DR5 TRAIL receptors which were not modified by treatment with GSI-I alone or GSI-I/TRAIL combined treatment (Fig. 2E). Moreover, no reduction in the level of the two splicing forms of c-FLIP nor pro-caspase- 8 cleavage was evidenced by western blot analysis (Fig. 2F).

GSI-I/TRAIL co-treatment induces a mitochondria-dependent apoptotic pathway. Then, we analysed whether treatment with the two drugs induces mitochondrial dysfunction in MDA-MB-231 cells. We evaluated the loss of mitochondrial transmembrane potential $(\Delta \psi \mathrm{m})$ by means of a flow cytometric assay which employs $\mathrm{DiOC}_{6}$, a mitochondrial specific and voltage-dependent dye. Fig. 3A shows that treatment of cells for $16 \mathrm{~h}$ with GSI-I or TRAIL employed separately caused only a weak dissipation of $\Delta \psi \mathrm{m}$ which instead reached about $65 \%$ after the GSI-I/TRAIL combined treatment.

This result focused the attention on a possible activation of caspase-9, the apoptotic protease activity related to mitochondrial dysfunction. Indeed, Fig. 3B shows that this enzyme was activated in MDA-MB-231 cells treated with the two drugs in combination, as indicated by the marked decrease in the procaspase form and the concomitant production of the active one at lower molecular weight. In the same conditions we also observed the activation of executioner caspase-3. None of these effects was observed by treating the cells with the two compounds employed separately.

The expression of apoptosis-related factors is modulated by treatment with GSI-I and/or GSI-I/TRAIL. It is well known that programmed cell death can be regulated by certain factors with either pro- or anti-apoptotic action. Thus, the upregulation of the former and/or the downregulation of the latter can be considered as key events in the induction of apoptosis (31). To this purpose, we examined the expression levels of some factors including Bcl-2 and IAP family members in MDA-MB-231 cells treated with GSI-I and/or TRAIL. As shown in Fig. 3C, we observed that the band corresponding to Noxa, a BH3-only member of Bcl-2 family, was undetectable in untreated cells while $1 \mu \mathrm{M}$ GSI-I treatment for $16 \mathrm{~h}$ significantly increased the intensity of the band (Fig. 3C). The increasing effect exerted by GSI-I was not modified by the addition of TRAIL. Moreover, the level of $\mathrm{Bim}_{\mathrm{S}}$ and $\mathrm{Bim}_{\mathrm{L}}$, two forms with major apoptotic action of the BH3-only factor Bim (32), also were increased after GSI-I or GSI-I/TRAIL treatment.

Moreover, we assessed the involvement of the anti-apoptotic factors $\mathrm{Bcl}-2, \mathrm{Bcl}-\mathrm{x}_{\mathrm{L}}$ and some IAP family proteins. The results reported in Fig. $3 \mathrm{C}$ indicate that in co-treated cells no variation on the levels of Bcl-2 and X-IAP occurred while the intensity of the bands corresponding to Bcl- $\mathrm{x}_{\mathrm{L}}$ and IAP-2 were clearly reduced. Only weak effects were observed on the c-IAP-1 level.

Survivin is another IAP family member, present in different alternative spliced forms, which is often overexpressed in various cancers (33). In MDA-MB-231 untreated cells, we detected the $37 \mathrm{kDa}$ form, endowed with high anti-apoptotic activity and the 2B form with lower anti-apoptotic action (34). The $37 \mathrm{kDa}$ band of survivin almost disappeared in cells treated with both GSI-I alone and in combination with TRAIL while the band corresponding to $2 \mathrm{~B}$ form increased (Fig. 3C). We hypothesize that the increase in this form could represent an attempt of the cells to counteract the cytotoxic effects induced by the drugs.

Many authors reported alterations in PI3K-AKT pathway in human cancers. Constitutively active PI3K or AKT results in an enhanced protection against apoptotic insults, such as growth factor deprivation, UV irradiation or loss of matrix attachment $(35,36)$. Moreover, it has been observed that in a large number of tumor cells the cytotoxic activity of anticancer drugs is accompanied by the downregulation of these enzymes (37). Thus, we analyzed in MDA-MB-231 cells the level of the active phosphorylated form of AKT and its possible variation following treatment with GSI-I and TRAIL, separately or in combination. Western blot analysis showed that the level of the band corresponding to phospho-AKT, measured by means of a specific antibody, dramatically decreased after treatment when the two drugs were combined (Fig. 3C).

Notch signaling knockdown promotes TRAIL-induced apoptosis. Since it has been demonstrated that $\gamma$-secretase inhibitors can act through different biochemical pathways, we investigated whether the synergistic effects induced by GSI-I/TRAIL combination were related to the specific inhibition of Notch signaling induced by GSI-I. We silenced the Notch gene and evaluated the effects of TRAIL addition. For these experiments we employed the sequence identified by Rizzo et al (26) which is able to knock down both Notch-1 and Notch-4 because of Notch-1, as demonstrated by the authors, is upstream of Notch-4. After confirming the reduction in Notch-1 level (Fig. 4A, upper panel), we analyzed the effect of TRAIL addition on MDA-MB-231 cell viability. Fig. 4B shows that treatment of Notch-1 silenced MDA-MB-231 cells with $25 \mathrm{ng} /$ $\mathrm{ml}$ TRAIL alone for $24 \mathrm{~h}$ induced cytotoxic effects very similar to those observed in non-silenced cells treated with GSI-I/ TRAIL combination. The results were also confirmed by light microscopy which evidenced the presence of the typical apoptotic morphology in TRAIL-treated siNotch-1 cells (Fig. 4C). Moreover, to confirm that the increase in the level of c-Jun 
A
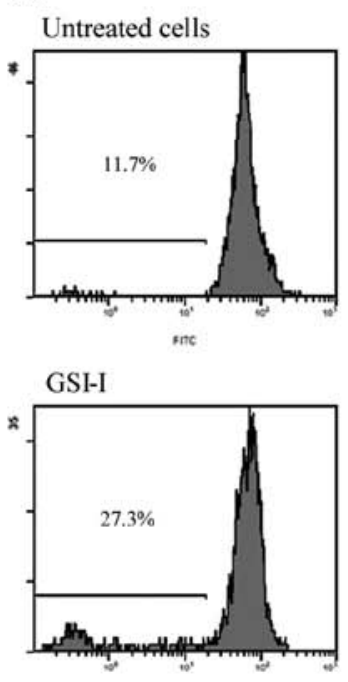

ninc
TRAIL

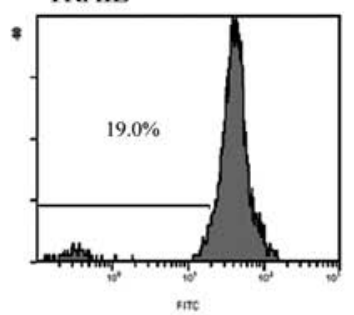

GSI-I/TRAIL

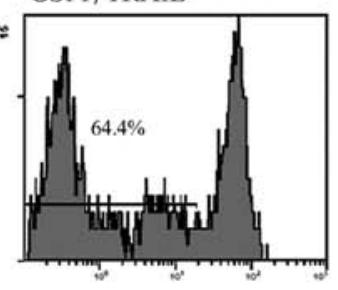

sec

B
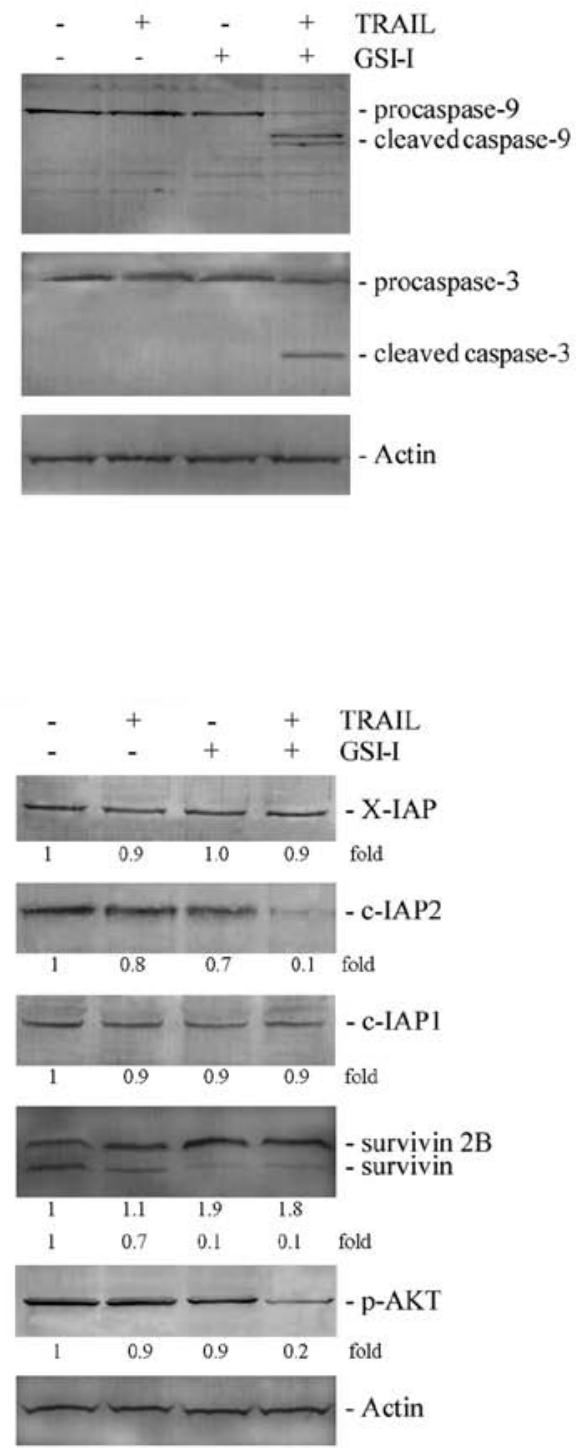

Figure 3. Cytotoxic effects of TRAIL and GSI-I are accompanied by dissipation of mitochondrial transmembrane potential ( $\Delta \psi \mathrm{m})(\mathrm{A})$, activation of caspase-9 and -3 (B) and modulation of pro- and anti-apoptotic factors (C). MDA-MB-231 cells were treated for $16 \mathrm{~h}$ in the presence of GSI-I and/or TRAIL. $\Delta \psi \mathrm{m}$ was evaluated by flow cytometry using the lipophilic dye $\mathrm{DiOC}_{6}$ as reported in Materials and methods and the levels of the proteins by western blot analysis using specific antibodies as indicated. Actin blots were included to show equal protein loading for all of the samples. Results are representative of three independent experiments.

and DR5 receptor were due to the downregulation of Notch induced by GSI-I we examined the levels of these factors in siNotch-1 silenced cells. As shown in Fig. 4A (lower panel) the basal levels of the bands corresponding to the two factors were markedly increased in siNotch-1 silenced cells. These effects were absent in scrambled siRNA transfected (siScr) cells.

\section{Discussion}

The aim of this study was to investigate the effect of GSI-I, one of the most frequently employed $\gamma$-secretase inhibitors, in modulating TRAIL sensitivity and activating apoptosis in TRAIL-resistant breast cancer cells. The hypothesis of associating TRAIL and GSIs arose by the observation that many tumor cells develop TRAIL resistance which can be overcome in the presence of different compounds. On the other hand, although displaying anticancer potential, GSIs often show toxic side-effects. For the first time data presented in this paper demonstrate a strong synergistic interaction between GSI-I and TRAIL which effectively reduced breast cancer cell viability also when employed at subtoxic doses (1 $\mu \mathrm{M}$ GSI-I/25 ng/ml TRAIL). Noteworthy, GSI-I/TRAIL combined treatment was particularly effective in MDA-MB-231 cells which are usually resistant to chemotherapy being ER-negative and mutated p53 (38). Differently, MCF-7 cells, another breast cancer cell line characterized by the presence of estrogen receptors and wild-type p53, were less sensitive to the effects of the combined treatment. 
A
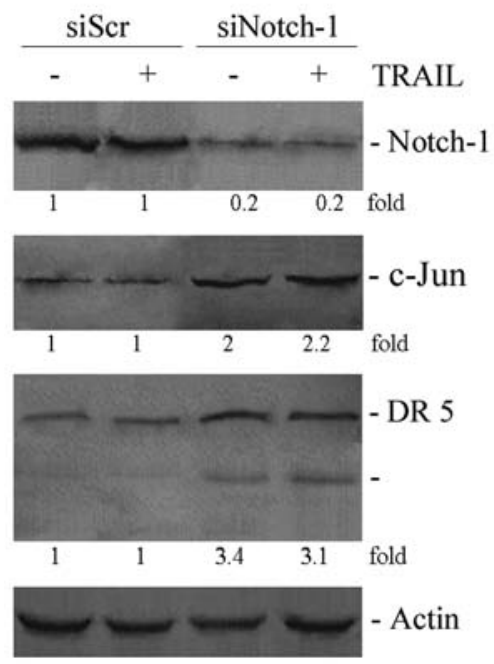

C
B

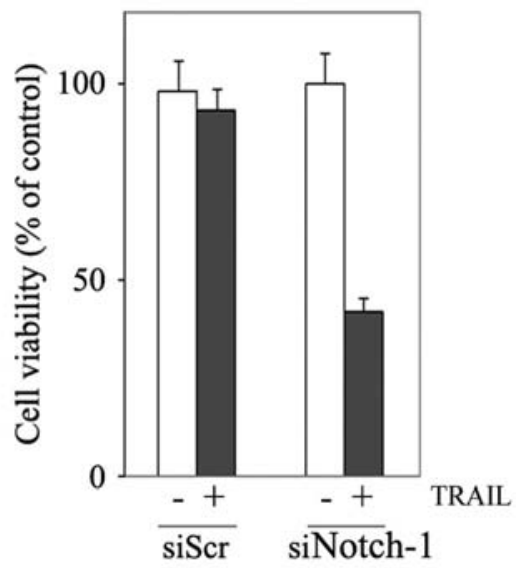

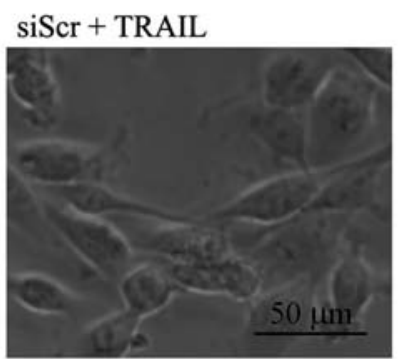

siNotch-1 + TRAIL

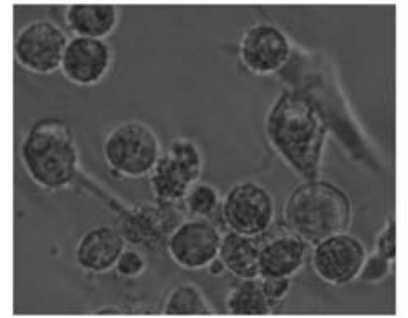

Figure 4. RNA interfering against Notch-1 in combination with TRAIL treatment mimics the cytotoxic effect of GSI-I/TRAIL association. MDA-MB-231 cells were transfected for $6 \mathrm{~h}$ with siNotch-1 or siScr. Cells were treated with $25 \mathrm{ng} / \mathrm{ml}$ TRAIL for $24 \mathrm{~h}$. (A) Western blot analysis of the levels of Notch-1, c-Jun and DR5 was performed as reported in Materials and methods. (B) Cell viability was estimated by MTT assay and expressed as the percentage of control value. (C) Apoptotic morphology of TRAIL-treated siNotch-1 silenced cells was evaluated by light microscopy, magnification, x200.

Morphological observations suggested that MDA-MB-231 cell death is related to the induction of apoptosis; cells were positive to Annexin V/propidium iodide test and the nuclei presented chromatin condensation. The activation of apoptotic pathway was also confirmed by the dissipation of transmembrane mitochondrial potential and the activation of caspase activities observed after combined treatment.

Many events induced by GSI-I could be responsible for sensitization of cells to TRAIL-mediated apoptosis and some of these are strictly related to the activity of GSI-I as inhibitor of $\gamma$-secretase, the main enzyme responsible for the cleavage and activation of Notch receptor (39). The results provided evidence that treatment with GSI-I strongly upregulates TRAIL receptors DR4 and DR5, at both mRNA and protein expression levels, an event which is involved in the sensitization to TRAIL-induced apoptosis by different compounds. Death receptor upregulation was associated with caspase- 8 activation and decrease in c-FLIP level, an anti-apoptotic protein which operates as an endogenous antagonist of caspase-8. Differently from MDA-MB-231 cells, MCF-7 cells are characterized by high levels of DR4 and DR5 receptors but the presence of c-FLIPL in an inhibitor complex with DR5, FADD and caspase- 8 make these cells resistant to TRAIL action (30). Data reported in this paper demonstrated that
GSI-I or GSI-I/TRAIL combined treatment was unable to modulate the expression level of TRAIL death receptors and of cFLIP in MCF-7 cells.

We were interested to ascertain the molecular mechanism whereby GSI-I enhances TRAIL receptor expression in MDA-MB-231 cells. It has been reported that the transcriptional activation of DR4 and DR5 can be dependent on the transcription factor AP1, a dimeric complex commonly constituted by c-Jun and c-Fos (29). In GSI-I-treated cells we observed an increase in level of c-Jun and in that of its phosphorylated active form, as well as, in the level of phospho-JNK, the enzyme responsible for c-Jun activation. Both these events can be related to Notch signaling; in fact, it has been reported that the active cytoplasmic fragment of Notch (NIC) inhibits AP1 (40) and, moreover, it can be responsible for the downregulation of JNK (41). We conclude that the failed cleavage of Notch consequent to the use of the $\gamma$-secretase inhibitor could prevent the inhibition of AP1 and JNK thus allowing the transcriptional activation of DR4 and DR5. However, since studies by Han et al suggested that GSIs can also act as inhibitors of proteasome activity (42), we can not exclude that the increased levels of DR4 and DR5 receptors were, al least partially, related to their failed degradation. 
The central role of Notch in MDA-MB-231 death pathway induced by GSI-I/TRAIL combination was also confirmed by silencing experiments against Notch. In siNotch silenced cells, we observed an increase in the levels of both c-Jun and DR5 similar to that observed after GSI-I or GSI-I/TRAIL treatment. Moreover, in silenced cells treated with TRAIL alone we also observed cytotoxic effects similar to those obtained after GSI-I/TRAIL combined treatment. Overall, data reported seem to indicate that the synergistic effects induced by the combination of the two drugs are a specific consequence of GSI-I action on $\gamma$-secretase activity.

Another mechanism through which GSI-I may sensitize MDA-MB-231 cells to apoptosis induced by TRAIL seems to be related to the modulation of pro- and anti-apoptotic factors. In many experimental models, the downregulation of survival factors and the concomitant upregulation of pro-apoptotic ones trigger a path that leads to cell death following treatment with anticancer drugs. Furthermore, it has been demonstrated a cross-talk between Notch signaling and the expression of some of these factors. Lee et al (43) reported in breast cancer cells the decrease of survivin as a consequence of Notch inhibition, while Rizzo et al reported that in breast cancer cells knockdown of Notch increased the levels of the pro-apoptotic factor Noxa (26). Data of the present study indicate that in MDA-MB-231 cells, besides the effects on Noxa and survivin, treatment with GSI-I/ TRAIL combination also induced a clear reduction in the levels of the phosphorylated form of AKT, which can be considered a key factor in growth and cell survival, together with that of c-IAP-2, another member of the IAP family. Moreover, some members of the Bcl-2 family, such as Bcl- $\mathrm{X}_{\mathrm{L}}$ and $\mathrm{Bim}_{\mathrm{EL}}$, are also downregulated following treatment with the two compounds in combination. Conversely, treatment with GSI-I and/or TRAIL involved upregulation of the proapoptotic factors $\mathrm{Bim}_{\mathrm{L}}$ and $\mathrm{Bim}_{\mathrm{S}}$.

In conclusion, the results presented here provide the first evidence that the inhibition of Notch signaling by GSI-I sensitizes MDA-MB-231 cells to TRAIL signaling, thus resulting in a strong synergistic antiproliferative effect on breast cancer cells. This is a consequence of: i) the marked increase in the expression level of DR4 and DR5, ii) the decrease of some survival factors and iii) the increase in some pro-apoptotic ones. These events, even if separated from each other, contribute to the induction of cell death. At first glance, our results seem to conflict with those reported by Wang et al (44) who demonstrated that in human hepatocarcinoma cells TRAIL-induced apoptosis can be triggered by the activation of Notch pathway. However, the authors indicated that the observed enhancement of TRAIL receptors is dependent on upregulation of p53 which is wild-type in hepatoma cells, differently from MDA-MB-231 breast cancer cells. Although the biochemical pathways induced by these compounds should be further explored, GSI-I/TRAIL association suggests a possible novel strategy for breast cancer treatment.

\section{Acknowledgements}

This study was supported by the University of Palermo (grants ORPA07EZ5Z and ORPA06F3TB).

\section{References}

1. Dotto GP: Notch tumor suppressor function. Oncogene 27: 5115-5123, 2008.

2. Fortini ME: Notch signaling: the core pathway and its posttranslational regulation. Dev Cell 16: 633-647, 2009.

3. Miele L, Miao H and Nickoloff BJ: Notch signaling as a novel cancer therapeutic target. Curr Cancer Drug Targets 6: 313-323, 2006.

4. Zardawi SJ, O'Toole SA, Sutherland RL and Musgrove EA: Dysregulation of Hedgehog, Wnt and Notch signaling pathways in breast cancer. Histol Histopathol 24: 385-398, 2009.

5. Sonoshita M, Aoki M, Fuwa H, Aoki K, Hosogi H, Sakai Y, Hashida H, Takabayashi A, Sasaki M, Robine S, Itoh K, Yoshioka K, Kakizaki F, Kitamura T, Oshima M and Taketo MM: Suppression of colon cancer metastasis by Aes through inhibition of Notch signaling. Cancer Cell 19: 125-137, 2011.

6. Wang Z, Li Y, Ahmad A, Banerjee S, Azmi AS, Kong D, Wojewoda C, Miele L and Sarkar FH: Down-regulation of Notch-1 is associated with Akt and FoxM1 in inducing cell growth inhibition and apoptosis in prostate cancer cells. J Cell Biochem 112: 78-88, 2011.

7. Bedogni B, Warneke JA, Nickoloff BJ, Giaccia AJ and Powell MB: Notch1 is an effector of Akt and hypoxia in melanoma development. J Clin Invest 118: 3660-3670, 2008.

8. Aster JC, Blacklow SC and Pear WS: Notch signaling in T-cell lymphoblastic leukaemia/ lymphoma and other haematological malignancies. J Pathol 223: 262-273, 2011.

9. Wang Z, Li Y, Banerjee S and Sarkar FH: Exploitation of the Notch signaling pathway as a novel target for cancer therapy. Anticancer Res 28: 3621-3630, 2008.

10. Bergmans BA and De Strooper B: Gamma-secretase: from cell biology to therapeutic strategies. Lancet Neurol 9: 215-226, 2010.

11. Panza F, Frisardi V, Imbimbo BP, Capurso C, Logroscino G, Sancarlo D, Seripa D, Vendemiale G, Pilotto A and Solfrizzi V: $\gamma$-secretase inhibitors for the treatment of Alzheimer's disease: the current state. CNS Neurosci Ther 16: 272-284, 2010.

12. Lin J, Zhang XM, Yang JC, Ye YB and Luo SQ: $\gamma$-secretase inhibitor-I enhances radiosensitivity of glioblastoma cell lines by depleting CD133+ tumor cells. Arch Med Res 41: 519-529, 2010.

13. Wang S and El-Deiry WS: TRAIL and apoptosis induction by TNF-family death receptors. Oncogene 22: 8628-8633, 2003.

14. Zhang XD, Nguyen T, Thomas WD, Sanders JE and Hersey P: Mechanisms of resistance of normal cells to TRAIL induced apoptosis vary between different cell types. FEBS Lett 482: 193-199, 2000.

15. Zhang L and Fang B: Mechanisms of resistance to TRAIL-induced apoptosis in cancer. Cancer Gene Ther 12: 228-237, 2005.

16. Kischkel FC, Lawrence DA, Chuntharapai A, Schow P, Kim KJ and Ashkenazi A: Apo2L/TRAIL-dependent recruitment of endogenous FADD and caspase- 8 to death receptors 4 and 5 . Immunity 12: 611-620, 2000.

17. Van Geelen CM, Pennarun B, Le PT, De Vries EG and De Jong S: Modulation of TRAIL resistance in colon carcinoma cells: different contributions of DR4 and DR5. BMC Cancer 11: 39-51, 2011.

18. Pellerito O, Calvaruso G, Portanova P, De Blasio A, Santulli A, Vento R, Tesoriere G and Giuliano M: The synthetic cannabinoid WIN 55,212-2 sensitizes hepatocellular carcinoma cells to tumor necrosis factor-related apoptosis-inducing ligand (TRAIL)-induced apoptosis by activating p8/CCAAT/enhancer binding protein homologous protein (CHOP)/death receptor 5 (DR5) axis. Mol Pharmacol 77: 854-863, 2010.

19. Kahana S, Finniss S, Cazacu S, Xiang C, Lee HK, Brodie S, Goldstein RS, Roitman V, Slavin S, Mikkelsen T and Brodie C: Proteasome inhibitors sensitize glioma cells and glioma stem cells to TRAIL-induced apoptosis by PKCE-dependent downregulation of AKT and XIAP expressions. Cell Signal 23: 1348-1357, 2011.

20. Lauricella M, Ciraolo A, Carlisi D, Vento R and Tesoriere G: SAHA/TRAIL combination induces detachment and anoikis of MDA-MB231 and MCF-7 breast cancer cells. Biochimie 94: 287-299, 2012.

21. Barten DM, Meredith Jr JE, Zaczek R, Houston JG and Albright CF: Gamma-secretase inhibitors for Alzheimer's disease: balancing efficacy and toxicity. Drugs RD 7: 87-97, 2006. 
22. Okuhashi Y, Itoh M, Nara N and Tohda S: Effects of combination of notch inhibitor plus hedgehog inhibitor or Wnt inhibitor on growth of leukemia cells. Anticancer Res 31: 893-896, 2011.

23. Chen F, Pisklakova A, Li M, Baz R, Sullivan DM and Nefedova Y: Gamma-secretase inhibitor enhances the cytotoxic effect of bortezomib in multiple myeloma. Cell Oncol (Dordr) 34: 545-551, 2011

24. Giuliano M, Pellerito O, Portanova P, Calvaruso G, Santulli A, De Blasio A, Vento R and Tesoriere G: Apoptosis induced in HepG2 cells by the synthetic cannabinoid WIN: involvement of the transcription factor PPARgamma. Biochimie 91: 457-465, 2009.

25. Calvaruso G, Giuliano M, Portanova P, Pellerito O, Vento R and Tesoriere G: Hsp72 controls bortezomib-induced HepG2 cell death via interaction with pro-apoptotic factors. Oncol Rep 18: 447-450, 2007.

26. Rizzo P, Miao H, D'Souza G, Osipo C, Song LL, Yun J, Zhao H, Mascarenhas J, Wyatt D, Antico G, Hao L, Yao K, Rajan P, Hicks C, Siziopikou K, Selvaggi S, Bashir A, Bhandari D, Marchese A, Lendahl U, Qin JZ, Tonetti DA, Albain K, Nickoloff BJ and Miele L: Cross-talk between Notch and the estrogen receptor in breast cancer suggest novel therapeutic approaches. Cancer Res 68: 5226-5235, 2008.

27. Chou TC and Talalay P: Quantitative analysis of dose effect relationship: the combined effect of multiple drugs or enzyme inhibitors. Adv Enzyme Regul 22: 27-55, 1984.

28. LeBlanc HN and Ashkenazi A: Apo2L/TRAIL and its death and decoy receptors. Cell Death Differ 10: 66-75, 2003.

29. Zou W, Liu X, Yue P, Zhou Z, Sporn MB, Lotan R, Khuri FR and Sun SY: c-Jun NH2-terminal kinase-mediated up-regulation of death receptor 5 contributes to induction of apoptosis by the novel synthetic triterpenoid methyl-2-cyano3,12-dioxooleana-1, 9-dien-28-oate in human lung cancer cells. Cancer Res 64: 7570-7578, 2004.

30. Day TW, Huang S and Safa AR: c-FLIP knockdown induces ligand-independent DR5-, -FADD-, caspase-8-, and caspase9-dependent apoptosis in breast cancer cells. Biochem Pharmacol 76: 1694-1704, 2008

31. Youle RJ and Strasser A: The BCL-2 protein family: opposing activities that mediate cell death. Nat Rev Mol Cell Biol 9 : 47-59, 2008

32. O'Connor L, Strasser A, O'Reilly LA, Hausmann G, Adams JM, Cory S and Huang DC: Bim: a novel member of the Bcl-2 family that promotes apoptosis. EMBO J 17: 384-395, 1998.
33. Li F: Role of survivin and its splice variants in tumorigenesis. Br J Cancer 92: 212-216, 2005.

34. Ryan B, O'Donovan N, Browne B, O'Shea C, Crown J, Hill AD, McDermott E, O'Higgins N and Duffy MJ: Expression of surviving and its splice variants surviving-2B and surviving-Ex 3 in breast cancer. Br J Cancer 92: 120-124, 2005.

35. Ghayad SE and Cohen PA: Inhibitors of the PI3K/Akt/mTOR pathway: new hope for breast cancer patients. Recent Pat Anticancer Drug Discov 5: 29-57, 2010.

36. Carnero A: The PKB/AKT pathway in cancer. Curr Pharm Des 16: 34-44, 2010.

37. Tenzer A, Zingg D, Rocha S, Hemmings B, Fabbro D, Glanzmann C, Schubiger PA, Bodis S and Pruschy M: The phosphatidylinositide 3'-kinase/Akt survival pathway is a target for the anticancer and radiosensitizing agent PKC412, an inhibitor of protein kinase C. Cancer Res 61: 8203-8210, 2001.

38. Chen JQ and Russo J: ERalpha-negative and triple negative breast cancer: molecular features and potential therapeutic approaches. Biochim Biophys Acta 1796: 162-175, 2009.

39. Borggrefe T and Oswald F: The Notch signaling pathway: transcriptional regulation at Notch target genes. Cell Mol Life Sci 66: 1631-1646, 2009.

40. Chu J, Jeffties S, Norton JE, Capobianco AJ and Bresnick EH Repression of activator protein-1 mediated transcriptional activation by the Notch-1 intracellular domain. J Biol Chem 277: 7587-7597, 2002

41. Kim JW, Kim MJ, Kim KJ, Yun HJ, Chae JS, Hwang SG Chang TS, Park HS, Lee KW, Han PL, Cho SG, Kim TW and Choi EJ: Notch interferes with the scaffold function of JNK-interacting protein 1 to inhibit the JNK signaling pathway. Proc Natl Acad Sci USA 102: 14308-14313, 2005.

42. Han J, Ma I, Hendzel MJ and Allalunis-Turner J: The cytotoxicity of gamma-secretase inhibitor I to breast cancer cells is mediated by proteasome inhibition, not by gamma-secretase inhibition. Breast Cancer Res 11: R57, 2009.

43. Lee CW, Raskett CM, Prudovsky I and Altieri DC: Molecular dependence of estrogen receptor-negative breast cancer on a notch-survivin signaling axis. Cancer Res 68: 5273-5281, 2008.

44. Wang C, Qi R, Li N, Wang Z, An H, Zhang Q, Yu Y and Cao X: Notch1 signaling sensitizes tumor necrosis factor-related apoptosis-inducing ligand-induced apoptosis in human hepatocellular carcinoma cells by inhibiting Akt/Hdm2-mediated p53 degradation and up-regulating p53-dependent DR5 expression. J Biol Chem 284: 16183-16190, 2009. 